\title{
Heisenberg Hamiltonian description of multiple-sublattice itinerant-electron systems: General considerations and applications to NiMnSb and MnAs
}

\author{
L. M. Sandratskii* \\ Max-Planck Institut für Mikrostrukturphysik, D-06120 Halle, Germany \\ R. Singer \\ Max-Planck Institut für Metallforschung, D-70569 Stuttgart, Germany
}

E. Şaşığlu

Institut für Festkörperforschung, Forschungszentrum Jülich, 52428 Jülich, Germany

(Received 20 May 2007; revised manuscript received 13 August 2007; published 7 November 2007)

\begin{abstract}
We consider magnetic systems where the magnetic sublattices can be unambiguously separated into sublattices of inducing and induced moments. The concrete numerical calculations are performed for half-metallic ferromagnetic Heusler compound NiMnSb and hexagonal phase of MnAs. In both systems, Mn atoms possess a robust atomic moment that is much larger than the induced moments of other atoms. It is shown that the treatment of the induced moments as independent variables of the Heisenberg Hamiltonian leads to artificial features in the spin-wave spectrum. We show that the artificial features of the model do not have a dramatic influence on the estimated value of the Curie temperature. This is demonstrated within both mean-field approximation and random-phase approximation. It is shown that the calculational scheme where the induced moments are assumed to fully adjust their values and directions to the adiabatic magnetic configurations of the inducing moments is free from the artificial feature in the spin-wave spectra. In this scheme, the exchange interaction between the inducing and induced moments appears as renormalization of the exchange interactions between inducing moments. It is shown that the redistribution of the exchange interactions has strong influence on the estimated value of the Curie temperature because of the decreased number of the degrees of freedom in the thermodynamic model. Different schemes of the mapping of the systems on the Heisenberg Hamiltonian are examined. The similarities and differences in the properties of NiMnSb and MnAs are discussed.
\end{abstract}

DOI: 10.1103/PhysRevB.76.184406

PACS number(s): 75.50.Cc, 75.30.Et, 71.15.Mb

\section{INTRODUCTION}

Historically, the first studies of the thermodynamics of itinerant-electron systems were performed within the framework of the Stoner theory where the effects of temperature are taken into account by means of the temperature dependence of the Fermi-Dirac distribution (see Ref. 1 for the overview of the theory of itinerant-electron magnetism). Although the Stoner picture is successful in the description of the ground-state properties of magnetic systems, the estimations of the Curie temperature for elementary $3 d$ ferromagnets exceed by about 1 order of magnitude the experimental values. The reason for the failure of the Stoner theory to describe the temperature dependences lies in the assumption that the vanishing of the net magnetization in the paramagnetic phase is the consequence of the vanishing of the local magnetization. The important low-energy magnetic excitations are missing in the Stoner theory that makes the decrease of the magnetization with heating too slow. These low-energy excitations are related to the transversal fluctuations of the magnetization and are routinely taken into account within the physical picture of localized atomic moments. The transversal fluctuations lead to the decrease of the net magnetization through the disordering of the atomic moments instead of their vanishing. Therefore, the features of the theory of localized atomic moments must be introduced into the theory of itinerant-electron magnets to describe the temperature effects and to estimate the Curie temperature.
At present, the main body of the studies of the thermodynamics of the itinerant-electron magnets is based on the mapping of the systems on a Heisenberg Hamiltonian (see, e.g., Refs. 2-8). The Heisenberg interatomic exchange parameters are calculated from the first principles on the basis of the density-functional theory. The knowledge of the exchange parameters allows the evaluation of the spin-wave dispersion, the magnetic transition temperature, the temperature dependence of magnetization, and other observable magnetic properties. In this way, the ab initio estimations of the Curie temperature of elementary $3 d$ magnets have been strongly improved. ${ }^{1}$

The procedure of the mapping of an itinerant-electron system on the Heisenberg Hamiltonian is based on an adiabatic hypothesis. ${ }^{5,9-11}$ Within this approach, the magnetic system is separated into subvolumes, "atoms." It is assumed that the directions of the magnetic moments of these areas, "atomic moments," can be considered as slow variables compared to the characteristic time of the relaxation of the electron states to a given set of directions. Note that the subvolumes are not necessary in the one-to-one correspondence to real atoms and can contain several or none of the atomic nuclei. ${ }^{11,12}$

The main focus of the given paper is on the itinerantelectron magnetic systems characterized by the property that different atomic sublattices play different roles in the magnetism of the materials. In many systems, it is possible to unambiguously distinguish the sublattices into, first, the sublattices with robust atomic magnetic moments formed prima- 
rily because of the intra-atomic exchange interaction and, second, the sublattices with induced moments that appear under the influence of the atomic moments of the sublattices of the first type.

Although the magnetism arises from the first type of sublattices, the properties of induced moments can influence importantly the physics of the material. For example, in a recent letter on half-metallic ferromagnet NiMnSb, Ležaić et $a l .{ }^{3}$ have demonstrated that the longitudinal fluctuation of the induced $\mathrm{Ni}$ moments being introduced into Heisenberg model leads to a singular temperature behavior of the magnetic susceptibility of the Ni sublattice. This singularity appears at the temperature that is much lower than the Curie temperature of the system. On the other hand, the conventional treatment of the Heisenberg model does not give any peculiarity in the temperature dependence of the magnetic susceptibility of the Ni sublattice.

Another example of the system where unusual properties of the sublattice of the induced moments has been recently found is hexagonal MnAs. ${ }^{13}$ Here, a nonmonotonous response of the induced As moments on the net magnetization of the Mn sublattice has been obtained. This property of the induced moments correlates with an instability of the collinear ferromagnetic structure of the Mn sublattice with respect to weak canting.

Further examples of the importance of the relation between inducing and induced moments are given by the following two physical problems many times discussed in the literature: the dependence of the interatomic exchange interaction between $3 d$ magnetic moments on the spin polarization of the $s p$ electrons (see, e.g., Ref. 14 and references therein), the strong noncollinearity between $s p$ and $d$ magnetization in strongly canted ferromagnets, ${ }^{15}$ and the magnetic ordering in diluted magnetic semiconductors by means of the formation of bound magnetic polarons. ${ }^{12}$ Finally, we would like to mention FeRh system where the properties of the induced $\mathrm{Rh}$ moment are responsible for the antiferromagnetic-ferromagnetic phase transition. ${ }^{16,17}$

In the present paper, we combine first-principles calculations and model-Hamiltonian treatment to address the questions related to the influence of the induced moments on the physics of magnetic materials. We discuss different approaches to the mapping of an itinerant-electron system on the Heisenberg Hamiltonian that can be considered as opposite limits in the treatment of the induced moments. We compare exchange parameters and Curie temperatures obtained within different schemes. We analyze NiMnSb and MnAs compounds revealing important differences and similarities between them.

\section{MAPPING OF THE ITINERANT ELECTRON SYSTEM ON A HEISENBERG HAMILTONIAN}

\section{A. General notes}

The mapping of an itinerant-electron system on a Heisenberg Hamiltonian

$$
H_{e f f}=-\sum_{\mu, \nu} \sum_{\substack{\mathbf{R}, \mathbf{R}^{\prime} \\\left(\mu \mathbf{R} \neq \nu \mathbf{R}^{\prime}\right)}} J_{\mathbf{R} \mathbf{R}^{\prime}}^{\mu \nu} \mathbf{e}_{\mathbf{R}}^{\mu} \mathbf{e}_{\mathbf{R}^{\prime}}^{\nu}
$$

consists in the determination of the interatomic exchange parameters $J_{\mathbf{R R}^{\prime}}^{\mu \nu}$ on the basis of first-principles densityfunctional-theory (DFT) calculations. In Eq. (1), the indices $\mu$ and $\nu$ number different sublattices, $\mathbf{R}$ and $\mathbf{R}^{\prime}$ are the lattice vectors specifying the atoms within sublattices, and $\mathbf{e}_{\mathbf{R}}^{\mu}$ is the unit vector pointing in the direction of the atomic magnetic moment at site $(\mu, \mathbf{R})$.

The physical fundamentals for the mapping is given by the adiabatic hypothesis that assumes that the variation of the directions of atomic moments under the influence of temperature or external magnetic field is slower than the adaptation of the electronic system to the given set of directions. Therefore, the directions of the magnetic moments, $\mathbf{e}_{\mathbf{R}}^{\mu}$, can be treated as classical parameters, whereas the energy of the system for each set of parameters, $E\left(\left\{\mathbf{e}_{\mathbf{R}}^{\mu}\right\}\right)$, is obtained in quantum-mechanical DFT calculations. The successful mapping on the Heisenberg Hamiltonian should give the parameters $J_{\mathbf{R R}^{\prime}}^{\mu \nu}$ that reproduce well the total-energy function $E\left(\left\{\mathbf{e}_{\mathbf{R}}^{\mu}\right\}\right)$.

Any concrete numerical calculation of the exchange parameters deals with the evaluation of the total energies of a certain subset of magnetic configurations $\left\{\mathbf{e}_{\mathrm{R}}^{\nu}\right\}$. Different calculational schemes use different subsets of the magnetic configurations. In an ideal Heisenberg system, the exchange parameters should not depend on the calculational procedure since the energy of any magnetic configuration is exactly described by Eq. (1) with the same set of the parameters. For real systems, the dependence on the method of calculation can, however, be strong. Below, we briefly characterize the main methods of the determination of the interatomic exchange parameters.

The most convenient and most frequently used method is suggested by the formula of Liechtenstein et al. ${ }^{2}$ The formula gives an analytical expression for the second derivatives of the band energy with respect to the deviations of the atomic moments. It is based on the magnetic force theorem that allows, to a certain accuracy, replacing the comparison of the total energies of the magnetic configurations by the comparison of the band energies. The exchange-correlation potential of the excited state is not calculated selfconsistently but is generated by the spinor rotation of the ground-state potential.

The second approach is based on the total-energy calculations for a set of collinear magnetic structures obtained by the reversal of the directions of some of the atomic moments. These calculations can be performed with any standard DFT code. They require, however, the use of large magnetic supercells. Since the change of the interspin angles obtained by reversal of the atomic moments is as large as $180^{\circ}$, the corresponding states of the system have low statistical weight in the statistical mechanics of the thermal magnetic disordering.

In this paper, we use frozen-magnon approach. It is more time consuming than the calculation with the formula of Liechtenstein et al. The method allows, however, the use of 
magnetic configurations with arbitrary angles between atomic magnetic moments. Therefore, the dependence of the exchange parameters on the choice of the magnetic configurations whose energy is evaluated can be studied. The calculations can be performed both with the use of the force theorem and self-consistency. This allows the control of the accuracy of the force-theorem calculations.

\section{B. Frozen-magnon approach}

The frozen magnon of the $\nu$ th sublattice characterized by wave vector $\mathbf{q}$ is defined by the expression

$\mathbf{e}_{\mathbf{R}}^{\nu}=\left[\sin \theta^{\nu} \cos \left(\phi^{\nu}(\mathbf{q})+\mathbf{q} \mathbf{R}\right), \sin \theta^{\nu} \sin \left(\phi^{\nu}(\mathbf{q})+\mathbf{q R}\right), \cos \theta^{\nu}\right]$,

where $\theta^{\nu}$ is the polar angle of the frozen magnon and $\phi^{\nu}$ is the initial phase angle of the sublattice that can be different for different $\mathbf{q}$.

Substituting Eq. (2) into Eq. (1), we get for the total energy

$$
\begin{aligned}
E(\mathbf{q},\{\theta\},\{\phi\})= & -\sum_{\mu, \nu}\left\{\cos \theta_{\mu} \cos \theta_{\nu} J_{0}^{\mu \nu}\right. \\
& \left.+\sin \theta_{\mu} \sin \theta_{\nu} \Re J^{\mu \nu}\left(\mathbf{q}, \Delta \phi^{\mu \nu}(\mathbf{q})\right)\right\},
\end{aligned}
$$

where

$$
\begin{gathered}
J^{\mu \nu}\left(\mathbf{q}, \Delta \phi^{\mu \nu}(\mathbf{q})\right)=\exp \left[-i \Delta \phi^{\mu \nu}(\mathbf{q})\right] \sum_{\mathbf{R}} \exp (i \mathbf{q} \mathbf{R}) J_{\mathbf{0}}^{\mu \nu}, \\
J_{0}^{\mu \nu}=\sum_{\mathbf{R}} J_{0 \mathbf{R}}^{\mu \nu}, \\
\Delta \phi^{\mu \nu}(\mathbf{q})=\phi^{\mu}(\mathbf{q})-\phi^{\nu}(\mathbf{q}) .
\end{gathered}
$$

In the case of $\Delta \phi^{\mu \nu}(\mathbf{q})=0$, we will use a simplified notation $J^{\mu \nu}(\mathbf{q}, 0) \equiv J^{\mu \nu}(\mathbf{q})$.

The evaluation of the exchange parameters involves several steps. To find the interaction parameters between the spins of the same sublattice $\nu$, we take a nonzero value of $\theta_{\nu}=\theta$. The polar angles of other sublattices are set to zero. The Heisenberg energy of such structures takes the form

$$
E^{\nu \nu}(\theta, \mathbf{q})=E_{0}^{\nu \nu}(\theta)-\sin ^{2} \theta J^{\nu \nu}(\mathbf{q}),
$$

where $E_{0}^{\nu \nu}(\theta)$ does not depend on q. Performing ab initio calculation of $E^{\nu \nu}(\theta, \mathbf{q})$ for a regular $\mathbf{q}$ mesh and making back Fourier transformation,

$$
J_{\mathbf{0}}^{\nu \nu}=\frac{1}{N} \sum_{\mathbf{q}} J^{\nu \nu}(\mathbf{q}) \exp (-i \mathbf{q} \mathbf{R}),
$$

we get the intrasublattice exchange parameters. Here, $N$ is the number of the points in the $\mathbf{q}$ mesh.

To find the exchange parameters for atoms belonging to two different sublattices $\nu$ and $\mu$, we take the nonzero value of the polar angle for these two sublattices: $\theta^{\nu}=\theta^{\mu}=\theta \neq 0$. The Heisenberg energy of such magnetic structure after some algebra can be represented in the form

$$
\begin{aligned}
E^{\mu \nu}\left(\theta, \mathbf{q}, \Delta \phi^{\mu \nu}(\mathbf{q})\right)= & E_{0}^{\mu \nu}(\theta)-\sin ^{2} \theta\left[J^{\nu \nu}(\mathbf{q})+J^{\mu \mu}(\mathbf{q})\right. \\
& +\exp \left(-i \Delta \phi^{\mu \nu}(\mathbf{q})\right) J^{\mu \nu}(\mathbf{q}) \\
& \left.+\exp \left(i \Delta \phi^{\mu \nu}(\mathbf{q})\right) J^{\mu \nu}(-\mathbf{q})\right],
\end{aligned}
$$

where $E_{0}^{\nu \mu}(\theta)$ is a $\mathbf{q}$-independent contribution to the energy. The single-sublattice terms $J^{\nu \nu}$ and $J^{\mu \mu}$ are known from the first step. To find the intersublattice exchange parameters, one needs to perform back Fourier transformation of $J^{\mu \nu}(\mathbf{q})$. In simple centrosymmetric two-sublattice systems, the quantities $J^{\mu \nu}(\mathbf{q})$ can be shown to be real. In this case, one $\mathbf{q}$ scan for given $\mu$ and $\nu$ is sufficient to determine the interatomic exchange parameters between the atoms of these sublattices. In general, however, $J^{\mu \nu}(\mathbf{q})$ are complex and the $a b$ initio calculations with two different phases $\Delta \phi^{\mu \nu}(\mathbf{q})$ are needed to fully determine $J^{\mu \nu}(\mathbf{q})$ on the basis of Eq. (9). The choice of these two phases can be different. For example, $\Delta \phi^{\mu \nu}(\mathbf{q})$ $=0$ and $\Delta \phi^{\mu \nu}(\mathbf{q})=\mathbf{q}\left(\mathbf{a}_{\mu}-\mathbf{a}_{\nu}\right)$ or $\Delta \phi^{\mu \nu}(\mathbf{q})=\mathbf{q}\left(\mathbf{a}_{\mu}-\mathbf{a}_{\nu}\right)$ and $\Delta \phi^{\mu \nu}(\mathbf{q})=\left(\mathbf{q}+\mathbf{K}_{m}\right)\left(\mathbf{a}_{\mu}-\mathbf{a}_{v}\right)$, where $\mathbf{K}_{m}$ is a vector of the reciprocal lattice.

\section{TWO-SUBLATTICE HEISENBERG MODEL: APPLICATION TO NiMnSb}

An important purpose of this paper is to contribute to the understanding of the properties of systems with different types of magnetic sublattices. This purpose is achieved by the combined consideration of both the first-principles calculations and the model-Hamiltonian treatment. In this and the next sections, the discussion is based on the results of the calculations for the half-metallic ferromagnet $\mathrm{NiMnSb}{ }^{18}$

The DFT calculations are performed with the augmented spherical wave (ASW) method ${ }^{19}$ within the generalized gradient approximation. The calculations of the spiral magnetic configurations [Eq. (2)] are based on the generalized Bloch theorem (see, e.g., Ref. 20) that allows, for an arbitrary wave vector $\mathbf{q}$, reducing the consideration of the magnetic spiral to the chemical unit cell of the crystal. The DFT calculation for the frozen-magnon states belongs to the type of the so-called constraint DFT calculations where the minimization of the total-energy functional is performed under restricting conditions. ${ }^{21}$

Most of the calculations reported in this paper are performed within the atomic sphere approximation. ${ }^{19}$ The constraint of the magnetic structure was imposed by choosing the directions of the local spin-quantization axes coinciding with the constrained directions of the atomic moments. The constraining fields ${ }^{21}$ orthogonal to the directions of magnetic moments were assumed to compensate the components of the atomic moments that are orthogonal to the directions of quantization. For the sake of comparison, a part of the calculations has been performed with a more time-consuming calculation based on the modification of the ASW method that takes into account the nonspherical part of the potential within the atomic spheres ${ }^{22}$ and calculates the constraining fields self-consistently.

The details of the crystal structure of Heusler compounds can be found, for example, in Ref. 23. The ground-state values of the spin moments of the $\mathrm{Mn}$ and $\mathrm{Ni}$ atoms were obtained to be $m_{\mathrm{Mn}}=3.85 \mu_{B}$ and $m_{\mathrm{Ni}}=0.20 \mu_{B}$. 


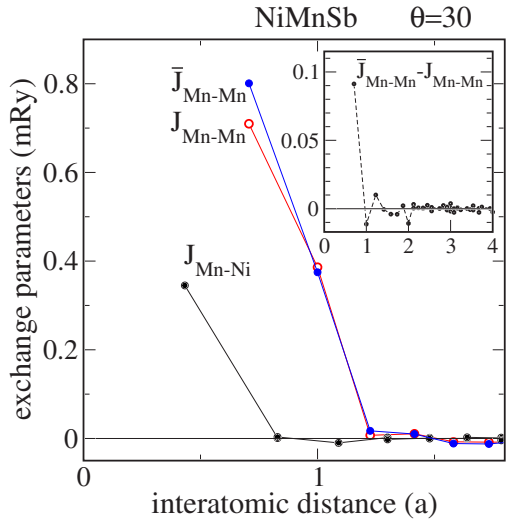

FIG. 1. (Color online) The calculated exchange parameters of NiMnSb. The parameters $J_{\mathrm{Mn}-\mathrm{Mn}}$ and $J_{\mathrm{Mn}-\mathrm{Ni}}$ are obtained with the use of the force theorem. The self-consistent SF2 scheme gives parameters that are very close to the results of the FT calculations. $\bar{J}_{\mathrm{Mn}-\mathrm{Mn}}$ presents the exchange parameters obtained with the use of the frozen-magnon energies calculated within the SF1 scheme. In the inset, the parameters $J_{\mathrm{Mn}-\mathrm{Mn}}$ and $\bar{J}_{\mathrm{Mn}-\mathrm{Mn}}$ are compared. All frozen-magnon calculations are performed for $\theta=30^{\circ}$. The exchange parameters are defined for discrete values of the interatomic distances. The lines connecting the values of the exchange parameters are guides for the eye.

In the following, we discuss the results of a two-sublattice frozen-magnon calculation for NiMnSb. The corresponding exchange parameters are shown in Fig. 1. Here, we consider $J_{\mathrm{Mn}-\mathrm{Mn}}$ and $J_{\mathrm{Mn}-\mathrm{Ni}}$ leaving the discussion of $\bar{J}_{\mathrm{Mn}-\mathrm{Mn}}$ until Sec. IV. The Ni-Ni exchange interactions are very weak and are not shown. In the following calculations, they are neglected.

The strongest exchange interaction takes place between the nearest and next-nearest $\mathrm{Mn}$ atoms and the nearest $\mathrm{Mn}$ and $\mathrm{Ni}$ atoms. Note that direct comparison of the Mn-Ni and Mn-Mn exchange parameters depicted in Fig. 1 overestimates the Mn-Ni exchange interaction because of the different numbers of the atoms in the $\mathrm{Ni}$ and $\mathrm{Mn}$ coordination spheres. The multiplication of the first $\mathrm{Mn}-\mathrm{Ni}$ and $\mathrm{Mn}-\mathrm{Mn}$ exchange parameters with the coordination numbers, respectively, 4 and 12 , gives the ratio of 0.16 . Therefore, the leading $\mathrm{Mn}-\mathrm{Ni}$ exchange, although weaker than the leading Mn-Mn exchange interaction, is significant. The following question arises: Does the account for the $\mathrm{Mn}-\mathrm{Ni}$ exchange interaction give the correction to the Curie temperature of the order of the ratio between the $\mathrm{Mn}-\mathrm{Ni}$ and $\mathrm{Mn}-\mathrm{Mn}$ exchange interactions? This question is addressed later in this section. First, we discuss the spin-wave excitations in systems with two inequivalent sublattices.

The spin-wave energies of such a system are given by the eigenvalues of the matrix

$$
\left(\begin{array}{cc}
J_{0}^{11}+J_{0}^{12}-J^{11}(\mathbf{q}) & -J^{12}(\mathbf{q}) \\
-J^{12}(\mathbf{q})^{*} & J_{0}^{12}
\end{array}\right)
$$

The eigenvectors of this matrix determine the shape of the spin waves. The analytical expression for the two spin-wave branches is given by formula

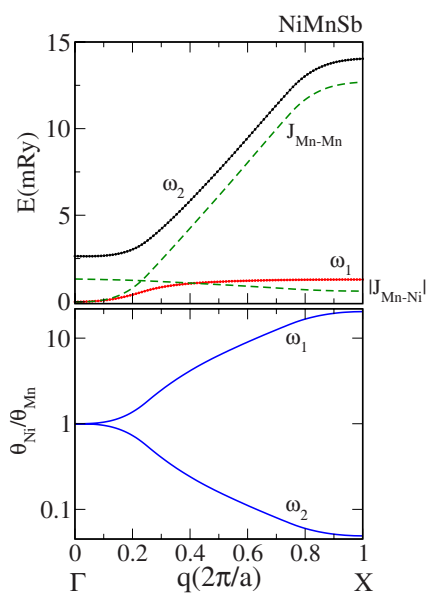

FIG. 2. (Color online) The properties of spin waves of $\mathrm{NiMnSb}$ calculated with the two-sublattice Heisenberg model. Upper panel: the spin-wave dispersions $\omega_{1,2}$. The broken curves present the $\mathbf{q}$ dependence of the exchange parameters. Lower panel: the ratio of the deviations of the atomic moments of $\mathrm{Mn}$ and Ni from the magnetization axis as a function of $\mathbf{q}$ and for both spin-wave branches. In the ordinate axis, the logarithmic scale is used.

$$
\begin{aligned}
\omega_{1,2}= & \frac{1}{2}\left[J_{0}^{11}-J^{11}(\mathbf{q})+2 J_{0}^{12}\right] \mp \frac{1}{2}\left\{\left[J_{0}^{11}-J^{11}(\mathbf{q})\right]^{2}\right. \\
& \left.+4\left|J^{12}(\mathbf{q})\right|^{2}\right\}^{1 / 2} .
\end{aligned}
$$

In Fig. 2, we show the spin-wave dispersion for $\Gamma X$ direction in the Brillouin zone. A notable feature of the spin-wave dispersion is the low-energy band that is weakly dispersive outside of a region around $\mathbf{q}=0$. To better understand the nature of the spin-wave bands, we consider the eigenvalues of matrix given by Eq. (10) in the case of $\left|J^{12}(\mathbf{q})\right| \ll \mid J_{0}^{11}$ $-J^{11}(\mathbf{q}) \mid$

$$
\begin{gathered}
\omega_{1}(\mathbf{q}) \approx J_{0}^{12}-\frac{\left|J^{12}(\mathbf{q})\right|^{2}}{J_{0}^{11}-J^{11}(\mathbf{q})} . \\
\omega_{2}(\mathbf{q}) \approx\left[J_{0}^{11}-J^{11}(\mathbf{q})\right]+J_{0}^{12}+\frac{\left|J^{12}(\mathbf{q})\right|^{2}}{J_{0}^{11}-J^{11}(\mathbf{q})} .
\end{gathered}
$$

We see that the first band is close to $J_{0}^{12}$, while the form of the second band is mainly determined by the term $\left[J_{0}^{11}\right.$ $\left.-J^{11}(\mathbf{q})\right]$ describing the spin-wave dispersion of the first sublattice in the case that intersublattice interaction is neglected. The eigenvectors of matrix (10) determine the ratio between deviation angles $\theta_{\mathrm{Mn}}$ and $\theta_{\mathrm{Ni}}$ in the corresponding spin-wave modes. This ratio as a function of $\mathbf{q}$ is presented for both bands in the lower panel of Fig. 2. In the logarithmic scale of the ordinate, the two curves are symmetric with respect to $\theta_{\mathrm{Mn}} / \theta_{\mathrm{Ni}}=1$. This means that for a given $\mathbf{q}$, the ratios for the two bands are reversed with respect to each other. At $\mathbf{q}=0$, the deviations are equal to each other for both acoustic and optic modes. For the acoustic mode, this simply means that the magnetic structure rotates as a whole that, in agreement with the Goldstone theorem, costs no energy. For larger $\mathbf{q}$, in 
the first spin-wave band, $\theta_{\mathrm{Ni}}$ is much bigger than $\theta_{\mathrm{Mn}}$, while the relation is opposite for the second band.

The appearance of the low-energy weakly dispersive magnon band is a characteristic feature of the theory where small induced moments are treated as independent adiabatic degrees of freedom (see, e.g., Ref. 24). A division of the unit cell into smaller pieces and an adiabatic treatment of the moments of these pieces lead to the appearance of additional spin-wave bands. ${ }^{11}$ Since there is no experimental evidence of the existence of these weakly dispersive low-energy bands, they should be considered as an artifact of the treatment of the induced moments.

The presence of the artificial features in the spin-wave spectrum raises the question of how far the treatment of the induced moments as independent Heisenberg variables influences the value of the Curie temperature. To answer this question, we consider the calculation of the Curie temperature of the two-sublattice model within the mean-field approximation (MFA) and random-phase-approximation (RPA).

In MFA, the Curie temperature is given by the largest eigenvalue of the matrix ${ }^{25}$

$$
\left(\begin{array}{cc}
J_{0}^{11} & J_{0}^{12} \\
J_{0}^{12} & 0
\end{array}\right)
$$

that takes the form

$$
k_{B} T_{C}^{M F A}=\frac{J_{0}^{11}}{3}\left\{1+\left[1+4\left(\frac{J_{0}^{12}}{J_{0}^{11}}\right)^{2}\right]^{1 / 2}\right\} .
$$

For $\left|J_{0}^{12}\right| \ll\left|J_{0}^{11}\right|$,

$$
T_{C}^{M F A} \approx \widetilde{T}_{C}^{M F A}\left[1+\left(\frac{J_{0}^{12}}{J_{0}^{11}}\right)^{2}\right]
$$

where $k_{B} \widetilde{T}_{C}^{M F A}=\frac{2}{3} J_{0}^{11}$ determines the Curie temperature in the case that only the interactions within the first sublattice are taken into account. As follows from Eq. (16), the correction to the Curie temperature because of the contribution from the induced sublattice is of the second order with respect to the intersublattice interaction. Indeed, the calculation of the $\mathrm{Cu}$ rie temperature of $\mathrm{NiMnSb}$ on the basis of Eq. (15) shows that it increases rather weakly from $1081 \mathrm{~K}$, in the case where the first sublattice only is considered, to $1101 \mathrm{~K}$, in the case that both $\mathrm{Mn}$ and $\mathrm{Ni}$ sublattices are taken into account.

Since an important feature of the RPA is the account for renormalized spin-wave excitations, ${ }^{26}$ it can be expected that the artificial features in the spin-wave dispersion will have here stronger influence on the Curie temperature than in MFA. In RPA, the Curie temperature of our model system is determined by the following two equations corresponding to the two sublattices considered: ${ }^{27}$

$$
\frac{1}{k_{B} T_{C}}=\frac{3}{2} \frac{1}{\Omega} \int \frac{d \mathbf{q}}{\left[J_{0}^{11}-J^{11}(\mathbf{q})\right]+\frac{m_{2}}{m_{1}} \frac{1}{J_{0}^{12}}\left[\left(J_{0}^{12}\right)^{2}-\left|J^{12}(\mathbf{q})\right|^{2}\right]},
$$

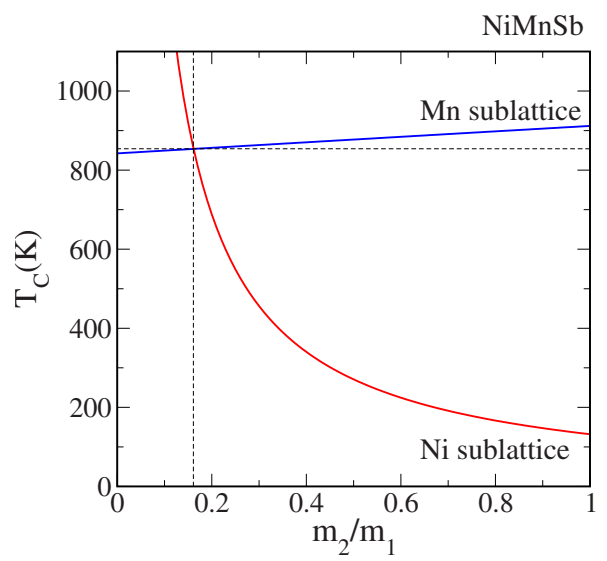

FIG. 3. (Color online) The graphical solution of the RPA equations [Eqs. (17) and (18)] for the Curie temperature of NiMnSb.

$$
\begin{aligned}
\frac{1}{k_{B} T_{C}}= & \frac{3}{2} \frac{m_{2}}{m_{1}} \frac{1}{J_{0}^{12}} \frac{1}{\Omega} \\
& \times \int \frac{\left\{\left[J_{0}^{11}-J^{11}(\mathbf{q})\right]+\frac{m_{2}}{m_{1}} J_{0}^{12}\right\} d \mathbf{q}}{\left[J_{0}^{11}-J^{11}(\mathbf{q})\right]+\frac{m_{2}}{m_{1}} \frac{1}{J_{0}^{12}}\left[\left(J_{0}^{12}\right)^{2}-\left|J^{12}(\mathbf{q})\right|^{2}\right]} .
\end{aligned}
$$

These equations determine simultaneously the Curie temperature and the limit of the ratio of the magnetizations of two sublattices, $\frac{m_{2}}{m_{1}}$, as the temperature tends to the Curie temperature. They can be solved by the variation of $\frac{m_{2}}{m_{1}}$ and searching for the intersection of the two curves $T_{C}\left(\frac{m_{2}}{m_{1}}\right)$ coming from both Eqs. (17) and (18).

It is instructive to compare Eq. (17) with the RPA expression for the Curie temperature in the one-sublattice system,

$$
\frac{1}{k_{B} T_{C}}=\frac{3}{2} \frac{1}{\Omega} \int \frac{d \mathbf{q}}{\left[J_{0}^{11}-J^{11}(\mathbf{q})\right]} .
$$

The difference of the two expressions is in the second term in the denominator that depends on the ratio of the sublattice magnetizations and on the exchange interaction between two sublattices. For small $\frac{m_{2}}{m_{1}}$, the Curie temperature of the twosublattice system should not differ much from the Curie temperature of the first sublattice.

The graphical solution of Eqs. (17) and (18) for the Mn and $\mathrm{Ni}$ sublattices of $\mathrm{NiMnSb}$ gives the ratio of the sublattice magnetizations about 0.16 (Fig. 3) that coincides with the relative strength of the $\mathrm{Mn}-\mathrm{Mn}$ and $\mathrm{Mn}-\mathrm{Ni}$ interactions estimated above.

Since we are working with reduced magnetizations, ${ }^{28}$ the ratio of the magnetizations at zero temperature is 1 . Therefore, the magnetization of the induced sublattice drops with heating faster than the magnetization of the inducing sublattice. As is seen from Eq. (17), this property diminishes the influence of the intersublattice exchange interaction on the Curie temperature and makes the correction of the Curie 
temperature small. For NiMnSb, the RPA- $T_{C}$ changes under the influence of the Ni sublattice from 842 to $854 \mathrm{~K}$ that gives a very small increase by $1.4 \%$.

Summarizing this part, we conclude that though the consideration of the induced moment as independent variable leads to artificial features in the magnon spectrum, this treatment does not influence dramatically the value of the Curie temperature. The effect of the $\mathrm{Mn}-\mathrm{Ni}$ exchange interaction on the Curie temperature is smaller than it might be expected from the relative value of the $\mathrm{Mn}-\mathrm{Mn}$ and $\mathrm{Mn}-\mathrm{Ni}$ exchange interactions. In the MFA formula, the change in the Curie temperature appears quadratic with respect to the ratio of the $\mathrm{Mn}-\mathrm{Ni}$ and $\mathrm{Mn}-\mathrm{Mn}$ exchange interactions, whereas in the RPA, the influence of the second sublattice is diminished by the small factor giving the ratio of the sublattice magnetizations.

It is important to understand how far the results obtained in this section depend on the procedure of the mapping of an itinerant-electron system on the Heisenberg Hamiltonian. In the following section, we develop another calculational scheme that is free from the artificial feature in the spin-wave spectrum discussed above. We show that the scheme results in significantly increased Curie temperature.

\section{RELAXATION OF THE INDUCED MOMENTS}

We assume now that only the moments of the inducing magnetic sublattice are independent adiabatic degrees of freedom. In this case, the total-energy function $E\left(\left\{\mathbf{e}_{i}\right\}\right)$ introduced above depends on the directions of the inducing moments only. In the DFT calculation of $E\left(\left\{\mathbf{e}_{i}\right\}\right)$, the directions of the inducing magnetic moments are constrained, whereas the induced moments relax freely within self-consistent Kohn-Sham procedure adjusting their sizes and directions to the constrained inducing moments. The size of the inducing moments is also determined self-consistently for each magnetic configuration. The force theorem in the form discussed by Liechtenstein $e t \mathrm{al}^{2}$ does not take into account the relaxation of the induced moments and cannot be applied here. Therefore, in the calculation of the exchange parameters, the total energies of the self-consistent states are used instead of the band energies of the non-self-consistent states employed in the force-theorem calculations.

Since we compare below the results of calculations within different schemes, it is convenient to introduce abbreviations simplifying the reference to the schemes. The non-self-

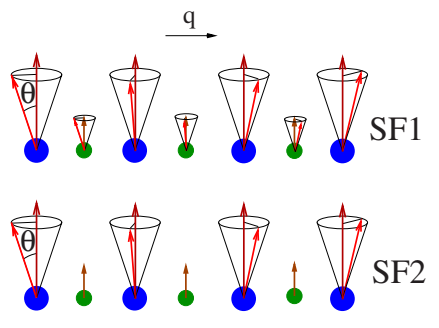

FIG. 4. (Color online) The schematic presentation of the SF1 (top) and SF2 (bottom) schemes for the self-consistent calculation of the frozen magnons. See text for the description.

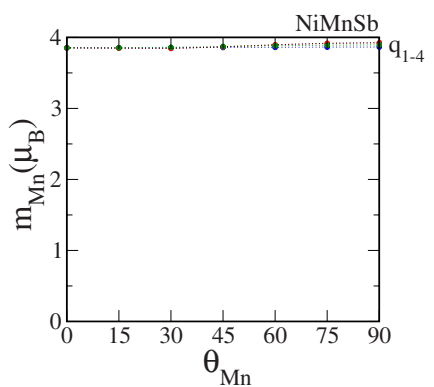

FIG. 5. (Color online) The Mn moment in NiMnSb as a function of $\theta_{\mathrm{Mn}}$. The calculations are performed with both SF1 and SF2 schemes and for various wave vectors q. The values of the Mn moment obtained in different calculations are close to each other, which reveals the robustness of the moment.

consistent force-theorem calculations will be referred to as FT. The self-consistent calculation with both the value and the direction of the induced moments to be relaxed will be referred to as SF1. The self-consistent calculation where the direction of the induced moments is kept parallel to the $z$ axis will be referred to as SF2. The directions of the magnetic moments used in SF2 calculation of the Mn-Mn exchange parameters are identical to the directions of the magnetic moments employed by the FT calculations. As pointed out above, the SF2 scheme uses the total energies obtained selfconsistently, while the FT employs the band part of the total energy obtained for the modified ground-state potential. The difference between SF1 and SF2 calculations is illustrated schematically in Fig. 4. The upper line of atoms presents the SF1 type of calculation where the induced moments selfconsistently adjust their directions to the directions of the inducing moments. In the lower line of atoms, the induced moments are kept parallel to the $z$ axis. In both cases, the magnetic structure of inducing sublattice is the same and is determined by the given $\theta$ and $\mathbf{q}$.

In Figs. 5-7, the results of the frozen-magnon calculations are presented for several selected wave vectors. The vectors are parallel to each other and differ by the length: $\mathbf{q}_{i}$ $=\left(0,0, \frac{i}{4}\right) \frac{2 \pi}{a}$. Here, $a$ is the lattice constant and $i=1,2,3,4$. The value of the wave vector determines the azimuthal

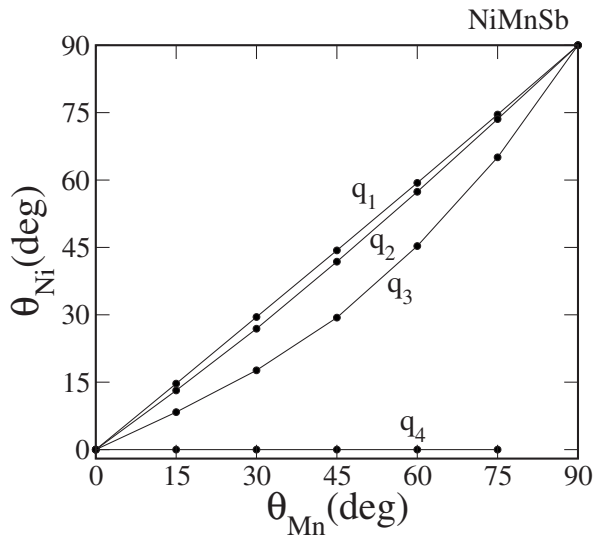

FIG. 6. $\theta_{\mathrm{Ni}}$ as a function of $\theta_{\mathrm{Mn}}$ for four different wave vectors of frozen magnons obtained within the SF1 calculation. 


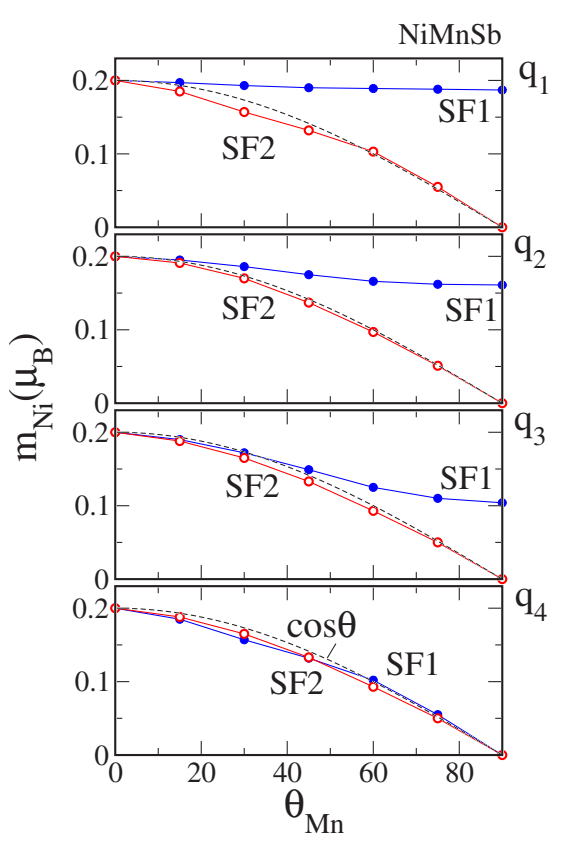

FIG. 7. (Color online) The induced Ni moment as a function of $\theta_{\mathrm{Mn}}$ for four different wave vectors of frozen magnons obtained within the SF1 and SF2 calculations. The broken curves present the $\cos \theta$-type dependence.

angles of the atomic moments [Eq. (1)]. For small q, the angles between neighboring $\mathrm{Mn}$ moments are small for any $\theta_{\mathrm{Mn}}$. On the other hand, for $\mathbf{q}=\mathbf{q}_{4}$, the local Mn magnetic structure depends strongly on $\theta_{\mathrm{Mn}}$ varying from the collinear ferromagnetism for $\theta_{\mathrm{Mn}}=0$ to collinear antiferromagnetism for $\theta_{\mathrm{Mn}}=90^{\circ}$.

Figure 5 shows the self-consistent $\mathrm{Mn}$ moment as a function of $\theta_{\mathrm{Mn}}$ calculated for frozen magnons with different wave vectors. The Mn moment is robust and depends weakly on the magnetic configuration that is in good agreement with the assumptions of the Heisenberg model of rigid atomic moments.

The properties of the induced Ni moments (Figs. 6 and 7) are very different. Figure 6 shows the angle $\theta_{\mathrm{Ni}}$ of the deviation of the Ni induced moment from the magnetization axis as a function of $\theta_{\mathrm{Mn}}$ obtained within the SF1 calculation. Since for small $\mathbf{q}$ the local structure of Mn moments is close to collinear ferromagnetism, the direction of the induced $\mathrm{Ni}$ moment is almost parallel to the directions of the surrounding Mn moments. Therefore, function $\theta_{\mathrm{Ni}}\left(\theta_{\mathrm{Mn}}\right)$ is almost linear. (For $\mathbf{q}=0, \theta_{\mathrm{Ni}}$ is exactly equal to $\theta_{\mathrm{Mn}}$.) With increasing $\mathbf{q}$, the deviation from collinearity increases that reflects increasing difference between $\theta_{\mathrm{Ni}}$ and $\theta_{\mathrm{Mn}}$. For $\mathbf{q}=\mathbf{q}_{4}$, the torques coming from different $\mathrm{Mn}$ atoms compensate and the induced $\mathrm{Ni}$ moments stay parallel to the $z$ axis independent of $\theta_{\mathrm{Mn}}$. [For $\mathbf{q}=\mathbf{q}_{4}$ and $\theta_{\mathrm{Mn}}=90^{\circ}$, the induced Ni moment is exactly zero (see Fig. 7 and the discussion below), and therefore an arbitrary $\theta_{\mathrm{Ni}}$ can be assigned at this point.]

The $\theta_{\mathrm{Mn}}$ dependence of the size of the induced Ni moments obtained within the SF1 scheme is also different for different $\mathbf{q}$. Since for small $\mathbf{q}$ the local configuration of $\mathrm{Mn}$ moments changes weakly with the variation of $\theta_{\mathrm{Mn}}$, the dependence of the value of the induced Ni moment on $\theta_{\mathrm{Mn}}$ is weak. With increasing $\mathbf{q}, m_{\mathrm{Ni}}$ as a function of $\theta_{\mathrm{Mn}}$ decreases faster. For $\mathbf{q}=\mathbf{q}_{4}$, the induced moment vanishes for $\theta_{\mathrm{Mn}}$ $=90^{\circ}$.

In contrast to the SF1 scheme, in the SF2 approach, the behavior of $m_{\mathrm{Ni}}\left(\theta_{\mathrm{Mn}}\right)$ is similar for all q values: $m_{\mathrm{Ni}}$ drops from the ground-state value at $\theta=0$ to zero at $\theta=90^{\circ}$. The dependence $m_{\mathrm{Ni}}\left(\theta_{\mathrm{Mn}}\right)$ is, in this case, well described by $\cos \theta_{\mathrm{Mn}}$, which means that $m_{\mathrm{Ni}}$ is approximately proportional to the $z$ projection of the Mn moments. For $\mathbf{q}=\mathbf{q}_{4}$, both SF1 and SF2 curves are very close to each other since in both cases the induced $\mathrm{Ni}$ moments are parallel to the $z$ axis. (These curves are not identical because of the properties of the induced $\mathrm{Sb}$ moments that we do not show.)

It is useful to relate the treatment of the $\mathrm{Ni}$ moment in $\mathrm{SF} 2$ scheme $\left(\mathbf{m}_{\mathrm{Ni}} \| O z\right)$ to the Stoner treatment of the temperature dependence of the magnetic moments. Similar to the Stoner model, in the SF2 scheme, the induced moments keep their directions parallel to the magnetization axis. The value of the Ni moment decreases because of the decrease of the net magnetization of the $\mathrm{Mn}$ sublattice. If we make the physical assumption that the direction of the induced moments is determined by the net moment of the whole $\mathrm{Mn}$ sublattice and not by the local Mn environment, the SF2 scheme is an adequate method to study the thermal processes in NiMnSb. In particular, the SF2 can be considered as proper technique to evaluate the Mn-Mn exchange parameters. The assumption that the induced moments keep to stay parallel to the $z$ axis with the disordering of the Mn moments is not supported by our SF1 calculations where the directions of the Ni moments are allowed to relax. Indeed, for small-q fluctuations of the Mn sublattice, the self-consistent Ni moments follow the directions of the neighboring Mn moments. It must, however, be noted that a decisive conclusion on which of the mapping schemes gives a better description of the physics of a given system may be made only on the basis of a more general calculational approach that contains both SF1 and SF2 schemes as special limiting cases. Such an approach should take into account longitudinal fluctuations of the moments as well as the dynamical properties of the fluctuations. The development of such approach is one of the complex problems of the solid state physics and is beyond the scope of the given paper. Instead, we use the comparison with experiment to draw some empirical conclusions.

On the next step, we compare the energies of the frozen magnons calculated with FT, SF1, and SF2 schemes.

In Fig. 8, we present the $\theta_{\mathrm{Mn}}$ dependences of the frozenmagnon energies. First, we notice that the difference between the curves obtained within SF1 and SF2 schemes is large for small $\mathbf{q}$ and decreases with increasing $\mathbf{q}$. This is in correlation with the properties of the induced Ni moments discussed above (Fig. 7): the difference in the values and directions of the induced moments obtained in two schemes is decreasing with increasing $\mathbf{q}$. The force-theorem calculations reproduce well the SF2 curves up to $\theta_{\mathrm{Mn}}$ of about $50^{\circ}$ for $\mathbf{q}_{1}$ and up to $75^{\circ}$ for $\mathbf{q}_{4}$.

Figure 1 presents the exchange parameters obtained with the frozen-magnon energies calculated for $\theta_{\mathrm{Mn}}=30^{\circ}$. The FT and SF2 schemes give similar values of the exchange parameters whose difference cannot be distinguished in the scale of Fig. 1. The main contributions into Mn-Mn exchange inter- 


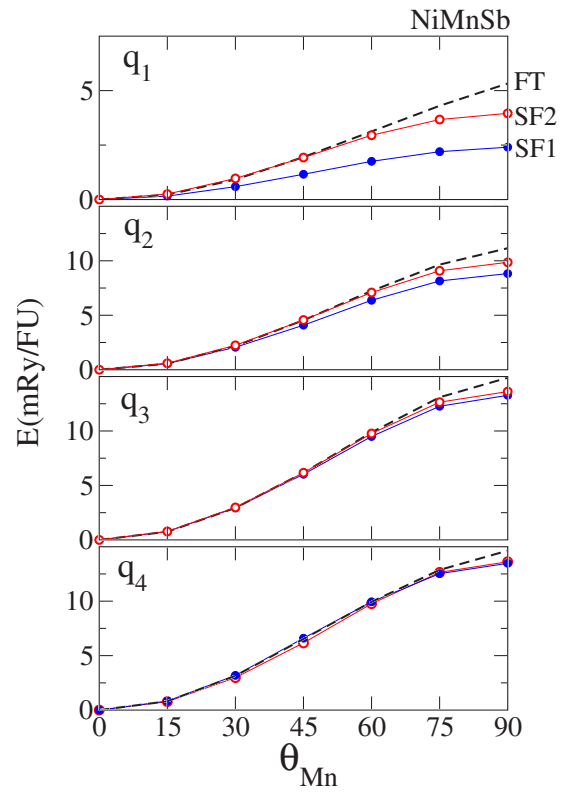

FIG. 8. (Color online) The frozen-magnon energy of NiMnSb as a function of $\theta_{\mathrm{Mn}}$ calculated with different schemes. The results are presented for four wave vectors. The broken curves give the results of the FT calculations. The curves with closed (open) circles are obtained within the SF1 (SF2) scheme.

actions give the interactions within two nearest coordination spheres. For Mn-Ni interaction, only the nearest neighbors contribute importantly. In Fig. 1, the exchange parameters are presented as a function of the interatomic distance. Distances assume discrete values corresponding to the radii of the coordination spheres. The lines are guides for the eye. The fast decay of the exchange parameters is related to the presence of the semiconducting gap in one of the spin channels of the half-metallic NiMnSb. ${ }^{6,29}$ For the comparison of the exchange parameters, it is important to take into account the number of the atoms in corresponding coordination spheres. For the Mn-Ni interaction, this number is 4, while for the two first Mn-Mn interactions, the coordination numbers are, respectively, 12 and 6 .

Analysis of Fig. 1 shows that the leading Mn-Mn exchange interaction obtained within the SF1 calculation of the frozen-magnon energies $(\bar{J})$ is significantly larger than the corresponding interaction obtained with the SF2 calculation. To reveal the origin of this difference in the exchange parameters, we consider the frozen-magnon energies as a function of $\mathbf{q}$ (Fig. 9). It is important to notice the change of the energy origin between Figs. 8 and 9. In Fig. 8, the origin is selected separately for each of the curves at the value of the energy at $\theta_{\mathrm{Mn}}=0$. On the other hand, in Fig. 9, the origin is the same for both curves and coincides with the ground-state energy.

The variation of the energy as a function of $\mathbf{q}$ is larger in the SF1 case (Fig. 9). At the right end of the q interval, the SF1 and SF2 energies are very close. This is expected since for this wave vector, the induced Ni moment is parallel to the $z$ axis in both schemes. The difference of the two curves stems from the region of small $\mathbf{q}$. At $\mathbf{q}=0$, the $S F 1$ structure coincides with the ground state, whereas in the SF2 calcula-

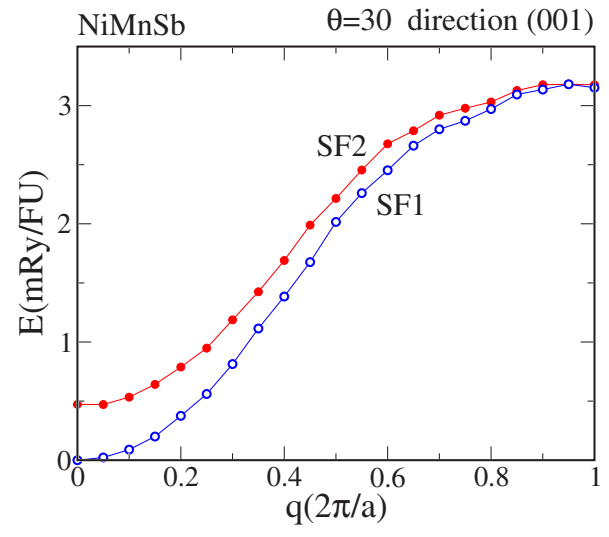

FIG. 9. (Color online) The frozen-magnon energies of NiMnSb as a function of $\mathbf{q}$ for the (001) direction of the Brillouin zone. The empty (filled) circles present the results of the SF1 (SF2) calculation. The calculations are performed for $\theta_{\mathrm{Mn}}=30^{\circ}$. To be compared with the spin-wave energies presented in Fig. 2, the frozen-magnon energies should be divided by $\sin ^{2} \theta$ [Eq. (7)] that for $\theta=30^{\circ}$ gives the multiplication factor of 4 .

tion, the $\mathrm{Ni}$ and $\mathrm{Mn}$ moments form angle $\theta_{\mathrm{Mn}}$. Therefore, the difference in the two curves is determined by the strength of the Mn-Ni exchange interaction.

In the previous section, both $\mathrm{Mn}$ and $\mathrm{Ni}$ atomic moments were treated as independent degrees of freedom. Respectively, the Mn-Ni exchange interaction is represented by a separate exchange parameter $J_{\mathrm{Mn}-\mathrm{Ni}}$. On the other hand, in the SF1 scheme, where only the Mn moments are treated as independent degrees of freedom, the $\mathrm{Mn}-\mathrm{Ni}$ exchange interaction appears as contribution to the renormalized $\mathrm{Mn}-\mathrm{Mn}$ exchange interaction. This explains why the leading Mn-Mn exchange parameter in the SF1 case is enhanced compared to the corresponding parameter in the SF2 scheme. The inset of Fig. 1 shows that significant change is obtained only for the exchange interaction between nearest $\mathrm{Mn}$ atoms.

As mentioned above, to compare the interatomic exchange interactions, the values of the exchange parameters must be multiplied by the corresponding coordination numbers that is by 4 for the Mn-Ni interaction and by 12 for the $\mathrm{Mn}-\mathrm{Mn}$ nearest neighbor interaction. Now, the contribution of the $\mathrm{Mn}-\mathrm{Ni}$ exchange interaction can be estimated as $12\left(\bar{J}_{\mathrm{Mn}-\mathrm{Mn}}-J_{\mathrm{Mn}-\mathrm{Mn}}\right) \approx 1.1 \mathrm{mRy}$ in the SF1 scheme and as $4 J_{\mathrm{Mn}-\mathrm{Ni}} \approx 1.4 \mathrm{mRy}$ in the scheme where the Ni moments are considered as independent variables. Both contributions of the $\mathrm{Mn}-\mathrm{Ni}$ exchange interaction are comparable. Therefore, the transition from one scheme to the other can be interpreted as different distribution of the Mn-Ni exchange interaction between exchange parameters.

This conclusion raises an important question: Does the redistribution of the exchange interactions influence the value of the Curie temperature? The answer to this question is positive. The change of the distribution of the exchange interactions is connected with the replacement of a twosublattice Heisenberg problem by a single-sublattice problem. The reduction of the number of the degrees of freedom changes the thermodynamic properties of the system. Indeed, we have seen above that in the two-sublattice case, a weak intersublattice interaction gives a second-order correction to 
TABLE I. Calculated Curie temperature (in K) of NiMnSb. The first row shows the exchange parameters used in the calculations. Three cases are considered. In the first case, only the unrenormalized Mn-Mn exchange parameters are used. In the second case, the $\mathrm{Ni}$ moments are considered as independent degrees of freedom. In the third case, the Mn-Mn exchange parameters renormalized because of the Mn-Ni exchange interaction are employed. Both the MFA and RPA estimations of the Curie temperature are presented.

\begin{tabular}{lccc}
\hline \hline & $J_{\mathrm{Mn}-\mathrm{Mn}}$ & $J_{\mathrm{Mn}-\mathrm{Mn}}, J_{\mathrm{Mn}-\mathrm{Ni}}$ & $\bar{J}_{\mathrm{Mn}-\mathrm{Mn}}$ \\
\hline MFA & 1081 & 1101 & 1212 \\
RPA & 838 & 858 & 973 \\
\hline \hline
\end{tabular}

the Curie temperature. On the other hand, the corresponding enhancement of the exchange Mn-Mn parameter in the onesublattice case gives the first-order correction to the Curie temperature and results in a substantially larger increase of $T_{C}$.

In Table I, we compare the values of the Curie temperatures obtained within different schemes on the basis of the frozen-magnon calculations for $\theta=30^{\circ}$. Indeed, the increase of $T_{C}$ due to the Mn-Ni interaction is stronger in the SF1 scheme where this interaction appears as a part of the renormalized Mn-Mn exchange parameters. All estimations exceed the experimental value of $730 \mathrm{~K}$. Therefore, on the present level of our knowledge, the SF2 scheme seems to provide a better basis for the study of the thermodynamics of the NiMnSb.

A useful information about the role of the exchange interaction between distant atoms is given by the Curie temperature calculated in the MFA as a function of the number of the coordination spheres taken into account (Fig. 10). The abscissa axis shows the maximal distance between Mn atoms whose interaction is included into the evaluation of the Curie temperature. The value of $T_{C}$ varies weakly when the included interatomic distances exceed $2 a$, where $a$ is the lattice parameter. Figure 10 also illustrates the similarity of the exchange parameters calculated with both force theorem (FT) and SF2 schemes.

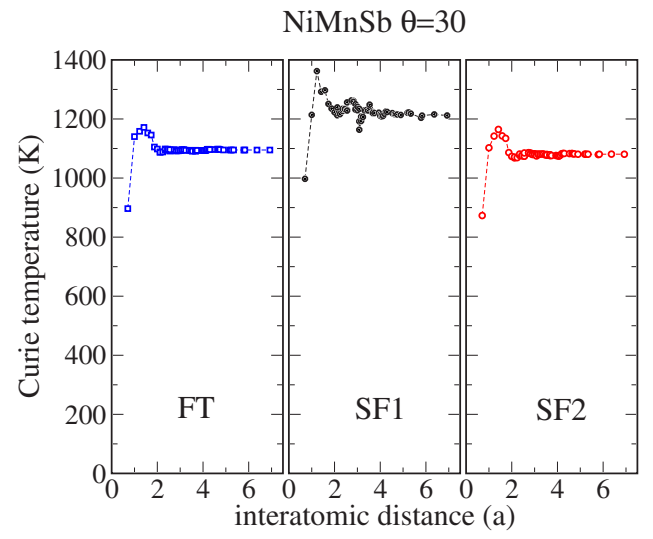

FIG. 10. (Color online) The Curie temperature of NiMnSb as a function of the maximal distance between $\mathrm{Mn}$ atoms whose interaction is taken into account. The Mn-Mn exchange parameters are calculated within the frozen-magnon approach with $\theta_{\mathrm{Mn}}=30^{\circ}$. Three calculational schemes are used: FT, SF1, and SF2.

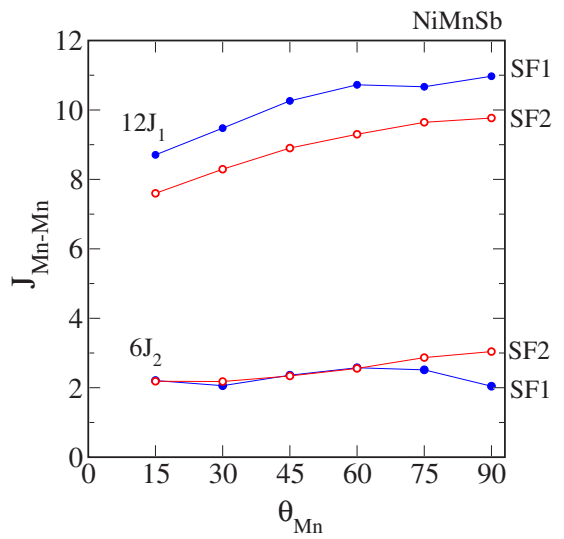

FIG. 11. (Color online) The dependence of the leading Mn-Mn exchange parameters on the angle $\theta_{\mathrm{Mn}}$ used in the frozen-magnon calculations. The parameters obtained within both SF1 and SF2 schemes are shown.

In an ideal Heisenberg system, the value of the Curie temperature does not depend on the value of the frozenmagnon angle $\theta$ used in the mapping procedure. For real itinerant-electron systems, the invariance of the Curie temperature with respect to the polar angle of the frozen magnons used in the calculations is not guaranteed and must be the subject of the study.

In Fig. 11, we compare the $\theta_{\mathrm{Mn}}$ dependence of first two Mn-Mn exchange parameters. In both SF1 and SF2 calculations, the leading exchange parameter between nearest Mn atoms increases substantially with increasing $\theta$.

In Fig. 12, we compare the Curie temperatures obtained with various schemes and for different values of the $\theta_{\mathrm{Mn}}$ in the frozen-magnon calculations. Similar to the calculation for $\theta_{\mathrm{Mn}}=30^{\circ}$, the SF2 and FT values of the Curie temperature are close to each other for the whole range of $\theta_{\mathrm{Mn}}$. The dependence of the estimated Curie temperature on $\theta_{\mathrm{Mn}}$ is

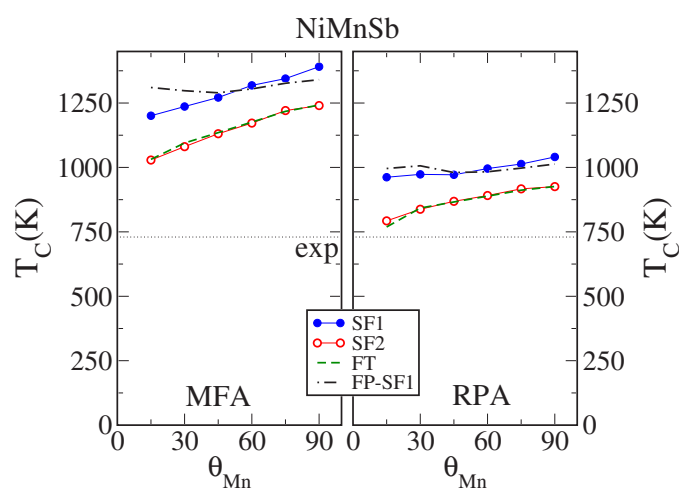

FIG. 12. (Color online) The calculated Curie temperature of $\mathrm{NiMnSb}$ as a function of the angle $\theta_{\mathrm{Mn}}$ used in the frozen-magnon calculations. The left panel presents the results of the mean-field calculations, whereas the results in the right panel are obtained within the random-phase approximation. In addition to the data obtained with the SF1, SF2 and FT schemes, we present the results of the SF1-type calculations that take into account the nonspherical part of the electron potential (these results are labeled as FP-SF1). The broken horizontal line at $730 \mathrm{~K}$ shows the experimental value of the Curie temperature. 
moderate. In the RPA calculations, it is weaker than in the MFA case. In full agreement with the conclusions formulated above, the SF1 scheme gives significant increase of the Curie temperature compared to the SF2 scheme. In addition to the FT, SF1, and SF2 schemes discussed earlier, we present the results of the calculation of the Curie temperature with the version of the ASW method that takes into account the nonspherical part of the potential within the atomic spheres (see the curves labeled FP-SF1 in the figure). ${ }^{22}$ The FP-SF1 data give weaker dependence of the MFA- $T_{C}$ on $\theta_{\mathrm{Mn}}$ than the SF1 data. In other aspects, the SF1 and FP-SF1 results are similar.

The comparison with the experimental value of the Curie temperature of $730 \mathrm{~K}$ shows that the theoretical values overestimate the experimental quantity. Therefore, at the present level of our knowledge, we should conclude that the Stonerlike treatment of the induced moment in $\mathrm{NiMnSb}$ gives a better description of $\mathrm{NiMnSb}$ than the assumption that the $\mathrm{Ni}$ moment quickly adjusts itself to the local Mn environment.

\section{MnAs}

Another system that we consider in this paper is hexagonal MnAs (see Ref. 13 and references therein). An unusual sequence of the phase transitions in MnAs attracts the attention of researches for many decades. Despite much efforts, the physics of the system remains the matter of debate. Recently, the studies on MnAs were further motivated by the possible applications in spintronic devices. Although the models suggested to explain the physics of hexagonal MnAs are very different in details, there is a general consensus that this system is characterized by an intimate connection between atomic structure and exchange interactions. In Ref. 13, we have shown that the hybridization of the $\mathrm{Mn}$ and As states leads in $\alpha$-MnAs to unusual nonmonotonous behavior of the induced As moment on the net magnetization of the Mn sublattice. The correlation between the unusual behavior of the induced moments and weak noncollinearity of the ground-state magnetic structure has been revealed.

Here, the scheme used in the study of $\mathrm{NiMnSb}$ in the previous sections is applied to hexagonal MnAs. In particular, we consider different treatments of induced As moments. The results obtained are compared with those for NiMnSb.

The angle of the deviation of the induced As moments from the $z$ axis as a function of the deviation angle of the Mn moments (SF1-type calculation) is presented in Fig. 13. The data are shown for three different values of the wave vector: $\mathbf{q}_{i}=\left(0,0, \frac{i}{3}\right) \frac{2 \pi}{c}$. Here, $c$ is the lattice parameter of the hexagonal lattice and $i=1,2,3$. In the main features, Fig. 13 is similar to the corresponding figure for NiMnSb (Fig. 6). Since the As moment is antiparallel to the Mn moment, $\theta_{\mathrm{As}}$ varies in the interval from $180^{\circ}$ to $90^{\circ}$ instead of the interval from $0^{\circ}$ to $90^{\circ}$ in NiMnSb. For $q=0$, the dependence is linear $\theta_{\mathrm{As}}=180^{\circ}-\theta_{\mathrm{Mn}}$, since in this case, the magnetic structure rotates rigidly. For small wave vector $\mathbf{q}_{1}$, the deviation of the curve $\theta_{\mathrm{As}}\left(\theta_{\mathrm{Mn}}\right)$ from linearity is weak. With increasing $\mathbf{q}$, the deviation increases. For $\mathbf{q}=\mathbf{q}_{3}$ corresponding to the azimuthal angle of $180^{\circ}$ between the moments of the nearest $\mathrm{Mn}$ atoms, the induced As moment keeps the direction parallel to the $z$ axis for any $\theta_{\mathrm{Mn}}$. (At $\mathbf{q}=\mathbf{q}_{3}$ and $\theta_{\mathrm{Mn}}=90^{\circ}$, the

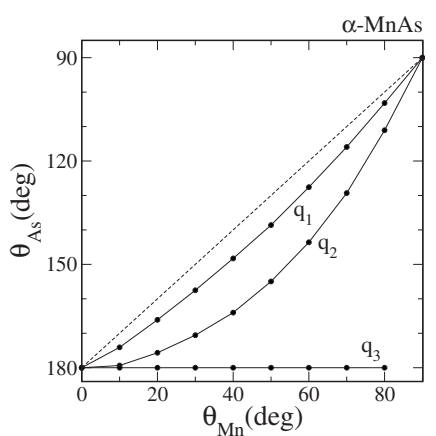

FIG. 13. $\theta_{\mathrm{As}}$ as a function of $\theta_{\mathrm{Mn}}$ for three different wave vectors of frozen magnons obtained within the SF1 calculation.

value of the induced As moment is exactly zero and, therefore, an arbitrary $\theta_{\text {As }}$ can be assigned at this point.)

The dependences of the values of atomic moments on $\theta_{\mathrm{Mn}}$ are presented in Fig. 14. Similar to the case of NiMnSb, the magnitude of the Mn moment depends weakly on the deviation angle. Therefore, $\mathrm{Mn}$ atoms possess a robust moment. On the other hand, the behavior of the induced As moment is unusual. Instead of decreasing with the decrease of the inducing net moment of the Mn sublattice, the induced moment increases for small $\theta_{\mathrm{Mn}}$ in both SF1 and SF2 calculations.

The peculiar behavior of the induced As moment correlates with an unexpected feature in the angular dependence of the total energy (Fig. 15). Both self-consistent calculations

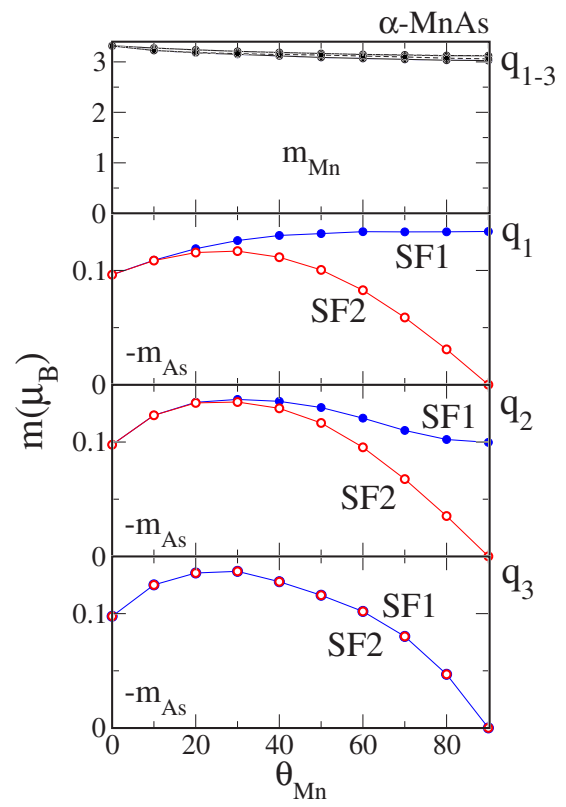

FIG. 14. (Color online) The Mn moment (upper panel) and induced As moment (three lower panels) as a function of $\theta_{\mathrm{Mn}}$ for three different wave vectors of frozen magnons. The calculations are performed within the SF1 and SF2 schemes. For Mn moment, all calculations give very close values that cannot be distinguished in the scale of the figure. The reversed value of the induced As moment is presented. The filled circles show the results of the SF1 calculation, whereas the empty circles are the results of the SF2 calculations. 


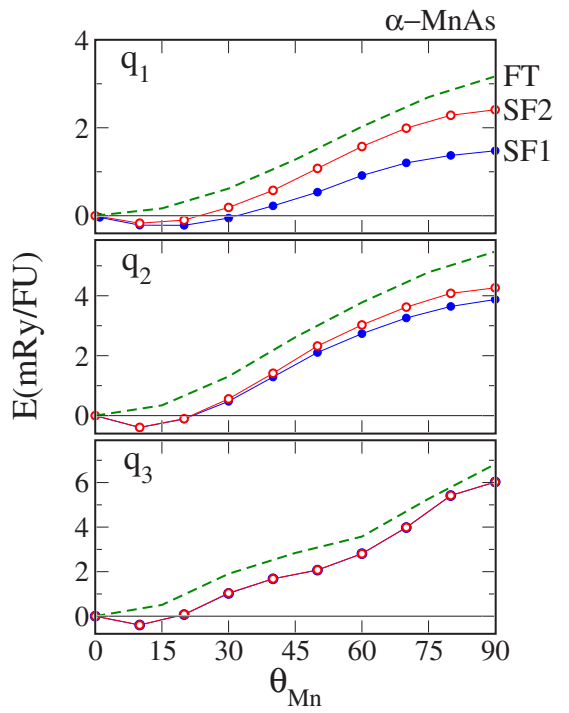

FIG. 15. (Color online) The frozen-magnon energy of MnAs as a function of $\theta_{\mathrm{Mn}}$ calculated with different schemes. The results are presented for three wave vectors. The broken curves give the results of the FT calculations. The curves with closed (open) circles are obtained within the SF1 (SF2) scheme.

give a decrease of the total energy for small $\theta_{\mathrm{Mn}}$ compared to the energy of the ferromagnetic state $\left(\theta_{\mathrm{Mn}}=0\right)$. On the other hand, the force-theorem calculations result in the behavior usual for a ferromagnetic system: the monotonous increase of the energy with increasing $\theta_{\mathrm{Mn}}$. The qualitative difference between the force-theorem and self-consistent data reveals the specific properties of the Mn-As hybridization in hexagonal MnAs that are the reason for the unusual physical behavior of this system.

The comparison of the SF1 and SF2 frozen-magnon energies (Fig. 15) leads to the conclusions similar to the conclusions drawn above for NiMnSb. For small q, the SF1 and $\mathrm{SF} 2$ curves differ strongly. The difference decreases with increasing $\mathbf{q}$ and disappears for $\mathbf{q}=\mathbf{q}_{3}$, since in the latter case, the induced As moments are parallel to the $z$ axis in both SF2 and SF1 calculations. The nonmonotonous dependence of the total energy on $\theta_{\mathrm{Mn}}$ cannot be described within a Heisenberg model.

The difference in the frozen-magnon energies obtained within different calculational schemes (Fig. 16) is reflected in the calculated exchange parameters. Indeed, we find significant increase in the first three exchange parameters (see inset of Fig. 16).

The consideration of the MFA- $T_{C}$ as a function of the number of the coordination spheres (Fig. 17) brings another unexpected result that is in strong contrast to the case of $\mathrm{NiMnSb}$ (Fig. 10). The convergence behavior of the Curie temperature appears to be very different for the two systems. In the FT calculation, $T_{C}$ saturates at the Mn-Mn distance of about $2 a$ similar to the case of NiMnSb. However, in the self-consistent calculations SF1 and SF2, the behavior is very different: A clear trend to the convergence of the Curie temperature is obtained at a much larger value of about $6 a$. In the SF1 scheme, the value of the Curie temperature drops from $626 \mathrm{~K}$ at $2.5 a$ to $433 \mathrm{~K}$ at $7 a$, which gives the decrease

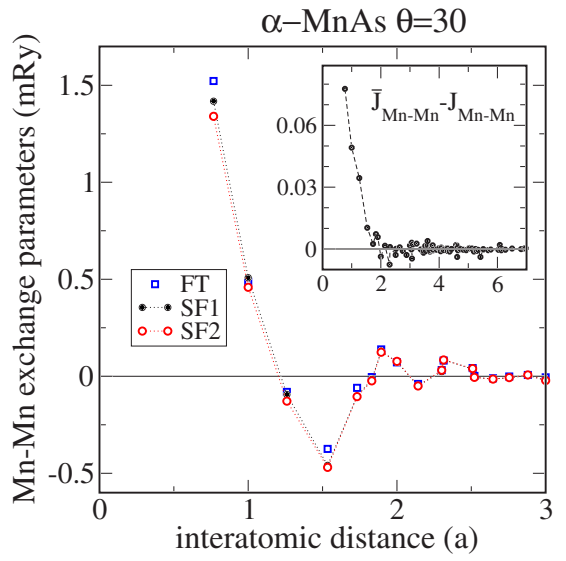

FIG. 16. (Color online) The calculated Mn-Mn exchange parameters of MnAs. The parameters are obtained within non-selfconsistent FT scheme and two self-consistent SF1 and SF2 schemes. The frozen-magnon approach has been used. Presented are the results for $\theta_{\mathrm{Mn}}=30^{\circ}$. The inset shows the difference between exchange parameters obtained within SF1 and SF2 schemes.

by $30 \%$. In the SF2 calculation, the decrease of the Curie temperature in the same interval takes place between 520 and $321 \mathrm{~K}$ and reaches the value of $38 \%$. Therefore, the selfconsistent account for the modification of the electron structure with the deviation of the atomic moments from the parallel directions increases the role of the longer-distance interatomic interactions. This is a specific feature of the $\alpha$-MnAs that is completely absent in the case of $\mathrm{NiMnSb}$ where the convergence of the Curie temperature with respect to the number of the coordination spheres taken into account does not depend on the calculational scheme (Fig. 10).

The Curie temperature as a function of $\theta_{\mathrm{Mn}}$ used in the evaluation of the exchange parameters is shown in Fig. 18. The force-theorem calculation gives rather weak dependence on $\theta_{\mathrm{Mn}}$. Therefore, $\alpha$-MnAs appears in the FT calculation as a good Heisenberg system. However, the values of the Curie temperature exceed strongly the experimental value of $T_{C}$ that is estimated to be about $400 \mathrm{~K}$. (Because of the magne-

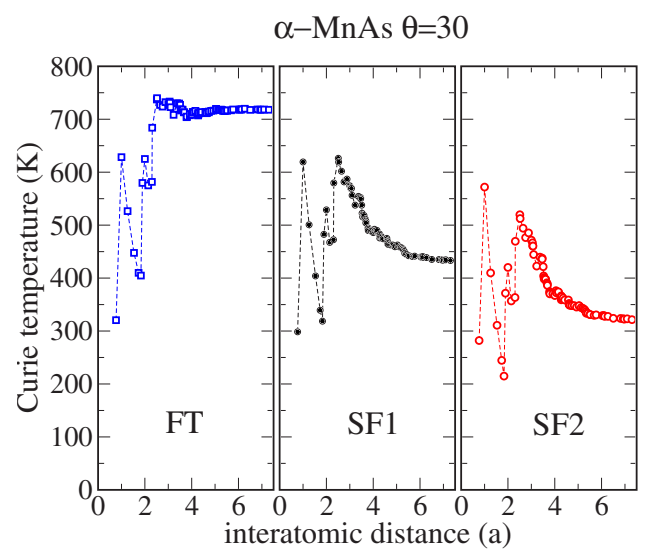

FIG. 17. (Color online) The Curie temperature of MnAs as a function of the maximal distance between Mn atoms whose interaction is taken into account. The Mn-Mn exchange parameters are calculated within the frozen-magnon approach with $\theta_{\mathrm{Mn}}=30^{\circ}$. Three different calculational schemes are used: FT, SF1, and SF2. 


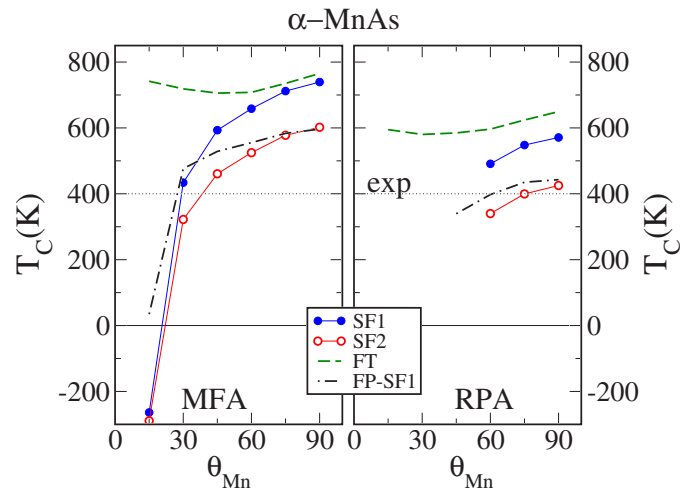

FIG. 18. (Color online) The calculated Curie temperature of MnAs as a function of the angle $\theta_{\mathrm{Mn}}$ used in the frozen-magnon calculations. The left panel presents the results of the mean-field calculations, whereas the results in the right panel are obtained within the random-phase approximations. In addition to the data obtained with the SF1, SF2 and FT schemes, we present the results of the SF1-type calculations that take into account the nonspherical part of the electron potential (these results are labeled as FP-SF1). The broken horizontal line at $400 \mathrm{~K}$ shows the experimental value of the Curie temperature obtained by the extrapolation of the magnetization as a function of temperature to the zero-magnetization point.

tostructural first-order phase transition at $313 \mathrm{~K}$, the intrinsic Curie temperature of the hexagonal MnAs is not reached experimentally. The extrapolation of the magnetization curve gives the value of about $400 \mathrm{~K}$.)

In the case of SF1 and SF2 calculations, the character of the $\theta_{\mathrm{Mn}}$ dependence of the Curie temperature is cardinally different. As a consequence of the canting instability, the low- $\theta_{\mathrm{Mn}}$ values of the exchange parameters underestimate the trend to the ferromagnetism. This is reflected in the negative values of the MFA estimations of the Curie temperature for $\theta_{\mathrm{Mn}}=15^{\circ}$ that reveals a strong contribution of the antiferromagnetic (negative) exchange parameters. The value of $T_{C}$ increases strongly with increasing $\theta_{\mathrm{Mn}}$. In the whole $\theta_{\mathrm{Mn}}$ interval, the SF1 and SF2 curves lie below the corresponding FT curves, giving the estimations of the $T_{C}$ that are closer to the experiment than the FT values.

In agreement with general theorems, MFA- $T_{C}$ is smaller than RPA- $T_{C}$. The reliable RPA estimations of the Curie temperature can be performed only for rather large values of $\theta_{\mathrm{Mn}}$. This is again the consequence of the weak canting of the magnetic ground state that leads to very small or even negative energies of some of the frozen magnons at small $\theta_{\mathrm{Mn}}$. The RPA formula for the Curie temperature, Eq. (19), contains the reversed energies of the spin-wave excitations, and the RPA value of $T_{C}$ is sensitive to the presence of such parts of the magnon dispersion. As the canting of the ground state is weak $\left(\approx 10^{\circ}\right)$, its influence becomes small for large $\theta_{\mathrm{Mn}}$ angles.

Similar to the case of NiMnSb, the values of the Curie temperature for SF1 calculation are significantly larger than the corresponding values for SF2 scheme. The reason for this is the same as in the case of NiMnSb: The Mn-Mn exchange parameters are enhanced by the Mn-As exchange interaction that appears in the SF1 scheme as a contribution to the renormalized Mn-Mn exchange interactions.
In Fig. 18, we also present the results of the calculations with the version of the ASW method that self-consistently takes into account the nonspherically symmetric part of the electronic potential in the atomic spheres. The calculations are performed within the SF1 type of scheme where the directions of the induced moments are fully relaxed to adjust themselves to the frozen-magnon configuration of the Mn moments (the results of this calculation are labeled with FPSF1 in Fig. 18).

The comparison with experimental estimation of the $\mathrm{Cu}-$ rie temperature shows that the best result is obtained within the most advanced FP-SF1 scheme where the RPA value of $T_{C}$ is close to the experimental $T_{C}$ for a broad interval of the $\theta_{\mathrm{Mn}}$ values.

Summarizing, $\alpha$-MnAs is the system with strong nonHeisenberg features that appear clearly if one goes beyond the force-theorem calculation of the interatomic exchange parameters. Concerning the treatment of the induced As moments in the calculation of the Mn-Mn exchange parameters, the best agreement with experiment is obtained within the scheme where the induced moments are assumed to adjust themselves to the adiabatic configurations of the Mn moments. In the case of $\mathrm{NiMnSb}$, we come to an opposite conclusion that a better agreement with experiment is obtained in the treatment where the induced moments were kept parallel to the magnetization axis.

\section{FURTHER COMMENTS}

In this section, we comment on the limitations of the calculational scheme and relate this work to the calculations by Mryasov performed for FePt and FeRh. ${ }^{16}$

\section{A. Limitations of the calculational scheme}

The theoretical scheme used in this paper does not rely on the use of adjustable parameters. A parameter-free description of multiple-sublattice systems is a complex physical and computational problem that cannot be solved without approximations. Below, we list some of the important limitations of our calculational scheme. Going beyond these limitations can provide other insights into the properties of magnetic systems with a significant role of induced moments.

First, the longitudinal fluctuations of the atomic moments are not taken into account. The values of the moments are calculated within a self-consistent procedure and characterize the lowest-energy state corresponding to a given magnetic configuration. The longitudinal fluctuations can be studied with, for instance, the use of the so-called fixed-spin-moment method (see, e.g., Ref. 30). The examples of the statistical mechanics calculations with account for longitudinal fluctuations are reported in Refs. 3, 31, and 32.

Second, it is desirable to complete the MFA and RPA calculations of the Curie temperature with Monte Carlo simulations. The Monte Carlo simulations although much more time consuming can, principally, provide an arbitrary accurate estimation of the Curie temperature for a given Hamiltonian. 
Third, in the calculation of the Curie temperature, the atomic moments are treated as classical quantities. A consistent account for quantum effects in the properties of atomic moments in solids is a very complicated problem for systems where the $3 d$ electrons hybridize strongly with the $s p$ electrons of the environment. In theories using the functional integral presentation of the free energy, the quantum effects can be taken into account by means of introducing random time-dependent fields and frequency dependent dynamic susceptibilities. ${ }^{33}$

\section{B. Comparison to Mryasov's study on FePt and FeRh}

It is useful to relate the calculational approach and physical conclusions of this paper to the studies of FePt and FeRh reported by Mryasov. ${ }^{16}$ There are important common points connecting the two works. Both works are dealing with systems where induced moments play a significant role and both use the noncollinear magnetic configurations to evaluate exchange interactions.

Mryasov performed calculations for two types of noncollinear magnetic structures. First, the magnetic moments within both $\mathrm{Fe}$ and $\mathrm{Pt} / \mathrm{Rh}$ sublattices were kept parallel, whereas the angle between the $\mathrm{Fe}$ and $\mathrm{Pt} / \mathrm{Rh}$ moments varied. The second type of the noncollinear configurations are plane spiral structures that correspond to angle $\theta=90^{\circ}$ in our notations $[\mathrm{Eq} .(2)]$. The calculations of the spirals were performed for one direction in the wave-vector space. The total energies were fitted with an effective Hamiltonian containing bilinear and biquadratic exchange interactions between $\mathrm{Fe}$ moments and the term depending on the length of the induced moment. The values and directions of the induced moments were determined by the effective Heisenberg-like intersublattice exchange fields. The intersublattice interaction of the Heisenberg type was not, however, included into the Hamiltonian. ${ }^{34}$ It was shown that the Hamiltonian can be rewritten in the form where the term depending on the value of the induced moments appears as contributions to the effective interaction between inducing Fe moments. The importance of the non-Heisenberg terms in the Hamiltonian is discussed.

The treatment of the independent degrees of freedom by Mryasov is similar to our treatment within the SF1 approach. Both works come to the conclusion that if the induced moments are not considered as independent degrees of freedom, their contribution to the energy of the system can be viewed as a modification of the exchange interactions between inducing atomic moments. Both studies establish the relation between the induced moments and the non-Heisenberg behavior of the systems.
The calculations of the spin-wave dispersion and Curie temperature of the $\mathrm{FePt} / \mathrm{FeRh}$ systems with different treatment of the induced moments are not reported in Ref. 16 and constitute an interesting problem for further application of the methodology used in this paper. An important difference between NiMnSb and MnAs studied here and FeRh is a very large induced moment on $\mathrm{Rh}$ that is close to $1 \mu_{B}$. The large value of the induced moment can have strong influence on the features of the fluctuation spectrum of the system. For the calculation of the Curie temperature, it is important to use the model Hamiltonian that describes the properties of the magnetic fluctuations with various directions of the wave vectors. In our work, this aim is achieved by the calculation for the spiral structures with the wave vectors sampling the whole Brillouin zone.

\section{CONCLUSIONS}

In this paper, we focus on the multiple-sublattice systems where the sublattices can be unambiguously separated into two different groups according to the ability to independently support their magnetization and, as a consequence, according to the role they play in the formation of the magnetization of the system. The study of the thermodynamics of complex itinerant-electron magnetic systems relies on the mapping of the systems on the model Hamiltonians. We show that different treatments of the induced moments in the mapping procedure lead to important qualitative and quantitative differences in the calculated magnetic properties. This conclusion is valid for systems that can be considered as good Heisenberg magnets as well as for the systems that show clear deviations from the Heisenberg behavior. We demonstrate that the use of the force theorem can lead in complex multisublattice systems to large errors in the calculated properties of the systems. For two systems considered, we suggest that for $\mathrm{NiMnSb}$, the consideration where the directions of the induced moments are kept parallel to the magnetization axis gives better agreement with experiment. On the other hand, for MnAs, the best agreement with experiment is obtained within the scheme where the directions of the induced moments adjust themselves to the adiabatic configuration of the Mn moments.

The consideration of a more general theory that contains the SF1 and SF2 schemes of treatment of induced moments as particular limiting cases is desirable. Such a theory should take into account the effects of dynamics and longitudinal fluctuations and help formulate a criterion for an a priori decision on the optimal treatment of the induced moments. In this respect, the main result of this paper is a contribution to the better formulation of the questions rather than providing final answers.

\footnotetext{
*1sandr@mpi-halle.de

${ }^{1} \mathrm{~J}$. Kübler, Theory of Itinerant Electron Magnetism (Clarendon, Oxford, 2000).

${ }^{2}$ A. I. Liechtenstein, M. I. Katsnelson, V. P. Antropov, and V. A.
}

Gubanov, J. Magn. Magn. Mater. 67, 65 (1987).

${ }^{3}$ M. Ležaić, Ph. Mavropoulos, J. Enkovaara, G. Bihlmayer, and S. Blügel, Phys. Rev. Lett. 97, 026404 (2006).

${ }^{4}$ E. Sasioglu, L. M. Sandratskii, and P. Bruno, Phys. Rev. B 70, 
024427 (2004).

${ }^{5}$ S. V. Halilov, H. Eschrig, A. Y. Perlov, and P. M. Oppeneer, Phys. Rev. B 58, 293 (1998).

${ }^{6}$ M. Pajda, J. Kudrnovsky, I. Turek, V. Drchal, and P. Bruno, Phys. Rev. B 64, 174402 (2001).

${ }^{7}$ L. Bergqvist, O. Eriksson, J. Kudrnovsky, V. Drchal, P. Korzhavyi, and I. Turek, Phys. Rev. Lett. 93, 137202 (2004).

${ }^{8}$ K. Sato, W. Schweika, P. H. Dederichs, and H. KatayamaYoshida, Phys. Rev. B 70, 201202(R) (2004).

${ }^{9}$ B. L. Gyorffy, A. J. Pindor, J. Staunton, G. M. Stocks, and H. Winter, J. Phys. F: Met. Phys. 15, 1337 (1985).

${ }^{10}$ V. P. Antropov, M. I. Katsnelson, B. N. Harmon, M. van Schilfgaarde, and D. Kusnezov, Phys. Rev. B 54, 1019 (1996).

${ }^{11}$ Q. Niu, X. Wang, L. Kleinman, W. M. Liu, D. M. C. Nicholson, and G. M. Stocks, Phys. Rev. Lett. 83, 207 (1999).

${ }^{12}$ J. M. D. Coey, M. Venkatesan, and C. B. Fitzgerald, Nat. Mater. 4, 173 (2005).

${ }^{13}$ L. M. Sandratskii and E. Sasioglu, Phys. Rev. B 74, 214422 (2006)

${ }^{14}$ E. Sasioglu, L. M. Sandratskii, and P. Bruno, Appl. Phys. Lett. 89, 222508 (2006).

${ }^{15}$ M. Fähnle, R. Singer, D. Steiauf, and V. P. Antropov, Phys. Rev. B 73, 172408 (2006).

${ }^{16}$ O. N. Mryasov, J. Magn. Magn. Mater. 272-276, 800 (2004); Phase Transitions 78, 197 (2005).

${ }^{17}$ M. E. Gruner, E. Hoffmann, and P. Entel, Phys. Rev. B 67, 064415 (2003).

${ }^{18}$ R. A. de Groot, F. M. Mueller, P. G. van Engen, and K. H. J. Buschow, Phys. Rev. Lett. 50, 2024 (1983).

${ }^{19}$ A. R. Williams, J. Kübler, and C. D. Gelatt, Phys. Rev. B 19, 6094 (1979).

${ }^{20}$ L. M. Sandratskii, Adv. Phys. 447, 91 (1998).

${ }^{21}$ P. H. Dederichs, S. Blügel, R. Zeller, and H. Akai, Phys. Rev.
Lett. 53, 2512 (1984).

${ }^{22}$ K. Knöpfle, L. M. Sandratskii, and J. Kübler, Phys. Rev. B 62, 5564 (2000).

${ }^{23}$ E. Sasioglu, L. M. Sandratskii, P. Bruno, and I. Galanakis, Phys. Rev. B 72, 184415 (2005).

${ }^{24}$ L. Udvardi, L. Szunyogh, K. Palotas, and P. Weinberger, Phys. Rev. B 68, 104436 (2003).

${ }^{25} \mathrm{P}$. W. Anderson, in Theory of Magnetic Exchange Interactions: Exchange in Insulators and Semiconductors, edited by F. Seitz and D. Turnbull, Solid State Physics Vol. 14 (Academic, New York, 1963), pp. 99-214.

${ }^{26} \mathrm{~S}$. V. Tyablikov, Methods of Quantum Theory of Magnetism (Plenum, New York, 1967).

${ }^{27}$ J. Rusz, I. Turek, and M. Diviš, Phys. Rev. B 71, 174408 (2005).

${ }^{28}$ The variables of the Heisenberg Hamiltonian (1) are chosen in the form of the unit vectors in the directions of the atomic moments.

${ }^{29}$ L. M. Sandratskii and P. Bruno, Phys. Rev. B 67, 214402 (2003).

${ }^{30}$ M. Uhl, L. M. Sandratskii, and J. Kübler, Phys. Rev. B 50, 291 (1994).

${ }^{31}$ J. Kübler, J. Phys.: Condens. Matter 18, 054402 (2007).

${ }^{32}$ A. V. Ruban, S. Khmelevskyi, P. Mohn, and B. Johansson, Phys. Rev. B 75, 054402 (2007).

${ }^{33}$ T. Moriya, Spin Fluctuations in Itinerant Electron Magnetism (Springer, Berlin, 1985).

${ }^{34}$ In this respect, the Hamiltonian of Mryasov differs from the Hamiltonians of Refs. 3, 31, and 32 where both the Heisenberg term and the term depending on the length of the moment are taken into account. Another important difference of the Mryasov's model compared to the models of Refs. 3, 31, and 32 is that the value of the induced moment is not an independent variable of the Hamiltonian and therefore the longitudinal fluctuations are not included into consideration. 